June 2013

\title{
Electroweak bremsstrahlung in bino-like dark matter annihilations
}

\author{
Kenta Shudo* and Takeshi Nihei ${ }^{\dagger}$ \\ Department of Physics, College of Science and Technology, \\ Nihon University, 1-8-14, Kanda-Surugadai, \\ Chiyoda-ku, Tokyo, 101-8308, Japan
}

\begin{abstract}
We investigate the effects of electroweak bremsstrahlung on bino-like neutralino dark matter pair annihilations in the minimal supersymmetric standard model (MSSM). We calculate the nonrelativistic pair annihilation cross sections via $W$-strahlung from leptonic final states, $\chi \chi \rightarrow W \ell \bar{\nu}$, and compare them with the contributions of the relevant two-body final states. We explore the case that sleptons lie below the $\mathrm{TeV}$ scale, while squarks are extremely heavy. It is found that the electroweak bremsstrahlung can give a dominant contribution to the cross section for some parameter regions which include slepton coannihilation regions with the observed relic abundance. We also evaluate the neutrino spectra at injection in the Sun. It is shown that energetic neutrinos via weak bremsstrahlung processes can be dominant over contributions of the two-body final states.
\end{abstract}

*Electronic address: shudo@phys.cst.nihon-u.ac.jp

${ }^{\dagger}$ Electronic address: nihei@phys.cst.nihon-u.ac.jp 


\section{INTRODUCTION}

Clarifying the nature of cold Dark Matter (DM) is one of the key issues in recent astrophysics and cosmology [1]. The relic abundance of cold DM in the present Universe is determined by recent astronomical observations with great precision as $\Omega_{\chi} h^{2}=0.1199 \pm 0.0027[2] .{ }^{1}$ Among the diverse candidates, the lightest superparticle (LSP) in supersymmetric models is one of the most attractive ones for the DM particle $[3,4]$. In the minimal supersymmetric standard model (MSSM) [5], the LSP is typically the lightest neutralino given by a linear combination of neutral gauginos and higgsinos

$$
\chi=\chi_{1}^{0}=N_{11} \tilde{B}+N_{12} \tilde{W}^{3}+N_{13} \tilde{H}_{1}^{0}+N_{14} \tilde{H}_{2}^{0},
$$

where $\tilde{B}$ is the $U(1)_{Y}$ gaugino (bino), $\tilde{W}^{3}$ is the neutral $S U(2)_{L}$ gaugino (wino), and $\tilde{H}_{1}^{0}$ and $\tilde{H}_{2}^{0}$ are the two neutral higgsinos with opposite hypercharges. The coefficients $N_{1 i}(i=1,2,3,4)$ are the elements of the $4 \times 4$ unitary matrix $N$ which diagonalizes the neutralino mass matrix $[5,6]$. Assuming the GUT relation for the gaugino masses, a bino-like LSP is realized for relatively light gauginos.

Cosmic rays produced by DM annihilations in the galactic halo provide a way of indirect detection of DM. For a bino-like LSP, the dominant annihilation channel is fermion pair production $\chi \chi \rightarrow f \bar{f}$. The neutralino pair annihilation cross section in the nonrelativistic limit is helicity suppressed $\left(\propto m_{f}^{2} / m_{\chi}^{2}\right)$ for light fermions due to the Majorana nature of the neutralino [7]. It is known, however, that gauge boson emissions can lift the helicity suppression, since the emitted gauge boson carries unit angular momentum. Indeed, it has been shown that the bremsstrahlung $\chi \chi \rightarrow f \bar{f} \gamma$ can potentially give characteristic signals of DM in gamma-ray observations $[8,9]$.

In recent years, the significance of electroweak bremsstrahlung emitting $W / Z$ bosons has been recognized in the literature [10-22]. In particular, the weak bremsstrahlung is expected to be more important in evaluating neutrino flux than the usual bremsstrahlung emitting photons, since the former emits primary neutrinos. The weak bremsstrahlung in a leptophillic dark matter model has been examined in Refs. $[10,11]$ where $\chi \chi \rightarrow W \ell \nu$ can give a dominant contribution over $\chi \chi \rightarrow \ell^{+} \ell^{-} \gamma$. The effect of the $W \ell \nu$ final states in this model is maximized in the limit where the dark matter mass is nearly degenerate with the mass of the $S U(2)_{L}$ doublet bosons

\footnotetext{
${ }^{1}$ The density parameter $\Omega_{\chi}=\rho_{\chi} / \rho_{c}$ is the DM energy density at present normalized by the critical density, and $h \approx 0.7$ is the Hubble constant in units of $100 \mathrm{~km} / \mathrm{sec} / \mathrm{Mpc}$.
} 
$\left(\eta^{0}\right.$ and $\left.\eta^{ \pm}\right)$which mediate the annihilation process similarly to sleptons. Gammaray signals in this model were investigated in Ref. [12]. Various signals, including positrons, were studied in Ref. [13]. Neutrino spectra including neutrino oscillation effects were explored in Ref. [14]. Helicity dependent effects on the neutrino spectra were studied for $S U(2)_{L}$ singlet Majorana fermion dark matter [15]. For a wino-like dark matter, initial state $W / Z$ radiations were found to be important [16]. Gamma rays from bino-like dark matter annihilations in the MSSM were examined including the three-body final state with weak bremsstrahlung [17]. Neutrino signals from weak bremsstrahlung in the MSSM were studied for a bino-like TeV dark matter scenario [18]. Antiproton constraints have been studied in Ref. [19] where effects of a longitudinal $W$-boson emission are examined in the presence of $S U(2)_{L}$ breaking effects $\left(m_{\eta^{0}} \neq m_{\eta^{ \pm}}\right)$, including the case of the constrained MSSM. The massive threebody final state, $W t b$, was considered in Ref. [20]. The role of weak bremsstrahlung for the relic density of DM was analyzed in Ref. [21].

In this paper, we investigate effects of electroweak bremsstrahlung on bino-like neutralino dark matter pair annihilations in the phenomenological MSSM where various SUSY parameters are chosen freely. We calculate the nonrelativistic pair annihilation cross sections via $W$-strahlung, $\chi \chi \rightarrow W \ell \bar{\nu}$, and compare them with the contributions of relevant two-body final states. We consider the case that squarks are extremely heavy ( $\gtrsim 10 \mathrm{TeV}$ ), while sleptons are much lighter than squarks. In this case, the weak bremsstrahlungs with quarks, $\chi \chi \rightarrow W u d, W c s, W t b$ via $t$ - and $u$-channel squark exchanges are suppressed. Then we discuss leptonic processes with primary neutrinos

$$
\chi \chi \rightarrow W^{+} \ell \bar{\nu}_{\ell}+\text { h.c. } \quad(\ell=e, \mu, \tau) .
$$

This paper is organized as follows. In Sec. II, we describe the relevant MSSM interactions. In Sec. III, we calculate the cross sections and neutrino spectra for the weak bremsstrahlung. In Sec. IV, we discuss the narrow width approximation for the massive weak bremsstrahlung process $\chi \chi \rightarrow W t b$. In Sec. V, we present our numerical results. Finally concluding remarks are given in Sec. VI. A simplified expression for the cross section in the unbroken $S U(2)_{L}$ limit in the slepton sector is provided in Appendix A. The contributions of two-body final states are listed in Appendix B. 


\section{RELEVANT MSSM INTERACTIONS}

The relevant interaction Lagrangian of the neutralino $\chi$ with leptons and sleptons can be written as

$$
\mathcal{L}_{\chi \ell \tilde{\ell}}=C_{L}^{(\nu)} \sum_{\ell} \bar{\chi} P_{L} \nu_{\ell} \tilde{\nu}_{\ell}^{*}+\sum_{\ell} \sum_{I=1,2} \bar{\chi}\left(C_{L I}^{(\ell)} P_{L}+C_{R I}^{(\ell)} P_{R}\right) \ell \tilde{\ell}_{I}^{*}+\text { h.c. },
$$

where $P_{L}=\frac{1-\gamma_{5}}{2}$ and $P_{R}=\frac{1+\gamma_{5}}{2}$. The fields $\tilde{\ell}_{I}(I=1,2)$ denote the charged slepton mass eigenstates, and $\tilde{\nu}_{\ell}$ is the sneutrino $(\ell=e, \mu, \tau)$. Flavor mixings and $\mathrm{CP}$ violations are neglected. The coupling constants in Eq. (3) are given by

$$
\begin{aligned}
& C_{L}^{(\nu)}=\frac{1}{\sqrt{2}}\left(-g N_{12}+g^{\prime} N_{11}\right), \\
& C_{L I}^{(\ell)}=\frac{1}{\sqrt{2}}\left(g N_{12}+g^{\prime} N_{11}\right)\left(\widetilde{V}_{\ell}\right)_{I 1}-h_{\ell} N_{13}\left(\widetilde{V}_{\ell}\right)_{I 2}, \\
& C_{R I}^{(\ell)}=-h_{\ell} N_{13}\left(\widetilde{V}_{\ell}\right)_{I 1}-\sqrt{2} g^{\prime} N_{11}\left(\widetilde{V}_{\ell}\right)_{I 2},
\end{aligned}
$$

where $g^{\prime}$ and $g$ denote the gauge coupling constant for the $U(1)_{Y}$ and $S U(2)_{L}, h_{\ell}=$ $g m_{\ell} /\left(\sqrt{2} m_{W} \cos \beta\right)$ is the Yukawa coupling constant for the lepton, and $N_{i j}$ is the element of the unitary matrix to diagonalize the neutralino mass matrix $[6,23]$. The vacuum angle $\beta$ is given by $\tan \beta=v_{2} / v_{1}$, where $v_{1}$ and $v_{2}$ are the vacuum expectation values of the two neutral Higgs bosons. The unitary matrix $\widetilde{V}_{\ell}$ diagonalizes the charged slepton mass squared matrix as $\widetilde{V}_{\ell} M_{\tilde{\ell}}^{2} \widetilde{V}_{\ell}^{\dagger}=\operatorname{diag}\left(m_{\tilde{\ell} 1}^{2}, m_{\tilde{\ell} 2}^{2}\right)$. The mass squared matrix, neglecting the $m_{\ell}^{2}$ terms, is approximately given by [6]

$$
M_{\tilde{\ell}}^{2}=\left(\begin{array}{cc}
m_{\tilde{\ell} L}^{2}+m_{Z}^{2}\left(-\frac{1}{2}+s_{W}^{2}\right) \cos 2 \beta & m_{\ell}\left(A_{\ell}-\mu \tan \beta\right) \\
m_{\ell}\left(A_{\ell}-\mu \tan \beta\right) & m_{\tilde{\ell} R}^{2}-m_{Z}^{2} s_{W}^{2} \cos 2 \beta
\end{array}\right),
$$

where $m_{\tilde{\ell} L}^{2}$ and $m_{\tilde{\ell} R}^{2}$ are the soft supersymmetry (SUSY) breaking mass parameters for the left- and right-handed sleptons, respectively, $s_{W}=\sin \theta_{W}$, and $A_{\ell}$ is the trilinear scalar coupling constant for the slepton. The mass eigenstates are related with the chiral bases $\tilde{\ell}_{L}$ and $\tilde{\ell}_{R}$ as $\tilde{\ell}_{I}=\left(\tilde{V}_{\ell}\right)_{I 1} \tilde{\ell}_{L}+\left(\tilde{V}_{\ell}\right)_{I 2} \tilde{\ell}_{R}$. The sneutrino mass squared is given by $m_{\tilde{\nu}}^{2}=m_{\tilde{\ell} L}^{2}+\frac{1}{2} m_{Z}^{2} \cos 2 \beta$. The $W$-boson emission from the slepton involves the following interaction:

$$
\mathcal{L}_{W \tilde{\ell} \tilde{\nu}}=-\sum_{\ell} \sum_{I=1,2} \frac{i g}{\sqrt{2}}\left(V_{\tilde{\ell}}\right)_{I 1}\left(\tilde{\ell}_{I}^{*} \stackrel{\leftrightarrow}{\partial^{\mu}} \tilde{\nu}\right) W_{\mu}^{-}+\text {h.c. }
$$


We follow the convention of Ref. [6] for the MSSM parameters.

We assume the GUT relation for the gaugino masses: $M_{1}=\frac{5}{3} M_{2} \tan ^{2} \theta_{W}$ where $M_{2}$ and $M_{1}$ are the gaugino masses for the $S U(2)_{L}$ and $U(1)_{Y}$ gauginos. Numerically, this implies $M_{1} \sim M_{2} / 2$. The neutralino in Eq. (1) is bino-like for $M_{1} \ll|\mu|$, where $\mu$ is the Higgsino mass parameter.

\section{CROSS SECTIONS FOR WEAK BREMSSTRAHLUNG}

In this section, we present the cross sections for the weak bremsstrahlung process (2) in the nonrelativistic limit $v \rightarrow 0$, where $v$ is the relative velocity between the two neutralinos in the center of mass frame.

The relevant Feynman diagrams for $\chi \chi \rightarrow W^{+} \ell \bar{\nu}_{\ell}$ via $t$ - and $u$-channel slepton exchange are shown in Fig. 1. The diagram A (B) proceeds via pair production $\chi \chi \rightarrow$ $\nu^{*} \bar{\nu}\left(\chi \chi \rightarrow \ell \bar{\ell}^{*}\right)$ followed by the $W$-boson emission from the neutrino (charged lepton). In the diagram $\mathrm{C}$, the $W$ boson is emitted by the virtual sleptons.

There exist other diagrams which can contribute to the process (2) in principle. However, in the parameter region we consider, they give only negligible effects. Contributions via $s$-channel $Z$-boson and pseudoscalar Higgs $(A)$ exchange $\chi \chi \rightarrow Z^{*} / A^{*}$ $\rightarrow \ell \bar{\ell}^{*} \rightarrow W \ell \bar{\nu}$ give no significant effects, since the neutralino coupling to the $Z$ boson or the pseudoscalar Higgs boson is highly suppressed for a bino-like LSP. ${ }^{2}$ Initial state radiation, in which an initial neutralino emits the $W$ boson, is negligible for a bino-like LSP, since a pure bino does not couple to a $W$ boson. Contributions via $W$-boson pair production followed by the $W$-boson decay to the leptonic pair, $\chi \chi \rightarrow$ $W W^{*} \rightarrow W \ell \bar{\nu}$, are negligible due to the suppressed coupling of the bino-like LSP to the $W$ boson. ${ }^{3}$

The 4-momentum of each particle in Fig. 1 is assigned as follows:

$$
\chi\left(k_{1}\right)+\chi\left(k_{2}\right) \rightarrow \ell\left(p_{1}\right)+\overline{\nu_{\ell}}\left(p_{2}\right)+W^{+}\left(p_{3}\right) .
$$

We define the sum of the $t$ - and $u$-channel diagram for each diagram $\mathrm{A}, \mathrm{B}$ and $\mathrm{C}$ in Fig. 1 as $\mathcal{M}_{\mathrm{A}}=\mathcal{M}_{\mathrm{A} t}+\mathcal{M}_{\mathrm{A} u}, \mathcal{M}_{\mathrm{B}}=\mathcal{M}_{\mathrm{B} t}+\mathcal{M}_{\mathrm{B} u}$ and $\mathcal{M}_{\mathrm{C}}=\mathcal{M}_{\mathrm{C} t}+\mathcal{M}_{\mathrm{C} u}$,

\footnotetext{
${ }^{2}$ Note, however, that a $s$-channel diagram is relevant for $t \bar{t}$ and $b \bar{b}$ final states, since the $t$ - and $u$-channel squark exchange diagrams are suppressed for extremely heavy squarks.

${ }^{3}$ This contribution is taken into account as a part of the two-body process $\chi \chi \rightarrow W W$ in Sec. V.
} 


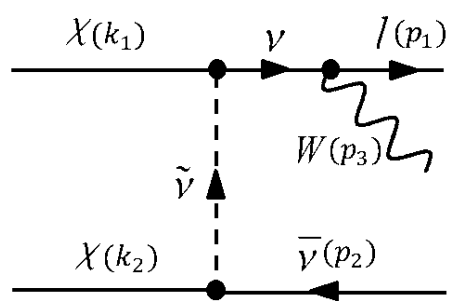

(A)

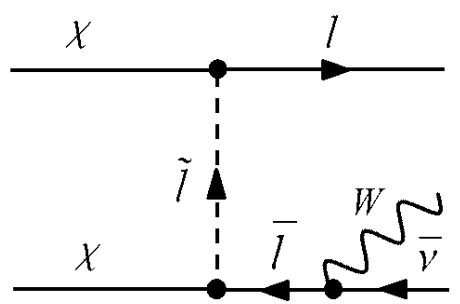

(B)

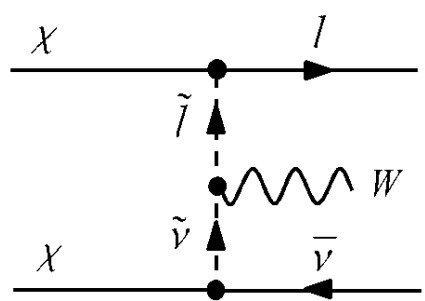

(c)

FIG. 1: Feynman diagrams for the weak bremsstrahlung process $\chi \chi \rightarrow W^{+} \bar{\nu}_{\ell} \ell$. The $W$ boson is emitted (A) from the virtual neutrino, (B) from the virtual charged lepton, and (C) from the virtual slepton line. Corresponding $u$-channel diagrams are not shown.

respectively. After the Fierz rearrangements, these matrix elements can be written as

$$
\begin{aligned}
\mathcal{M}_{\mathrm{A}}= & \frac{i g}{2 \sqrt{2}} \frac{1}{t_{2}-m_{\tilde{\nu}}^{2}} \frac{1}{q_{1}^{2}}\left(C_{L}^{(\nu)}\right)^{2}\left(\overline{u_{\ell}} \nexists_{3}^{*} \not q_{1} \gamma^{\alpha} P_{L} v_{\nu}\right)\left(\overline{v_{\chi}} \gamma_{\alpha} \gamma_{5} u_{\chi}\right) \\
\mathcal{M}_{\mathrm{B}}= & \sum_{I=1,2} \frac{i g}{2 \sqrt{2}} \frac{1}{t_{1}-m_{\tilde{\ell} I}^{2}} \frac{1}{q_{2}^{2}} C_{L I}^{(\ell)} \\
& \times\left[-C_{L I}^{(\ell)}\left(\overline{u_{\ell}} \gamma^{\alpha} \not \phi_{2} \nexists_{3}^{*} P_{L} v_{\nu}\right)\left(\overline{v_{\chi}} \gamma_{\alpha} \gamma_{5} u_{\chi}\right)+C_{R I}^{(\ell)}\left(\overline{u_{\ell}} \phi_{2} \nexists_{3}^{*} P_{L} v_{\nu}\right)\left(\overline{v_{\chi}} \gamma_{5} u_{\chi}\right)\right] \\
\mathcal{M}_{\mathrm{C}}= & \sum_{I=1,2} \frac{i g}{2 \sqrt{2}} \frac{1}{t_{2}-m_{\tilde{\nu}}^{2}} \frac{1}{t_{1}-m_{\tilde{\ell} I}^{2}}\left(V_{\tilde{\ell}}\right)_{I 1}\left[\varepsilon_{3}^{*} \cdot\left(p_{1}-p_{2}\right)\right] C_{L}^{(\nu)} \\
& \times\left[C_{L I}^{(\ell)}\left(\overline{u_{\ell}} \gamma^{\alpha} P_{L} v_{\nu}\right)\left(\overline{v_{\chi}} \gamma_{\alpha} \gamma_{5} u_{\chi}\right)-C_{R I}^{(\ell)}\left(\overline{u_{\ell}} P_{L} v_{\nu}\right)\left(\overline{v_{\chi}} \gamma_{5} u_{\chi}\right)\right]
\end{aligned}
$$

where $q_{1}=p_{1}+p_{3}, q_{2}=p_{2}+p_{3}$, and $\varepsilon_{3}=\varepsilon\left(p_{3}\right)$ is the polarization vector of the $W$ boson. The spinors $u$ and $v$ are denoted as $u_{\chi}=u\left(k_{1}\right), v_{\chi}=v\left(k_{2}\right), u_{\ell}=u\left(p_{1}\right), v_{\nu}$ $=v\left(p_{2}\right)$, where the spin indices are suppressed. The Lorentz invariants $t_{1}$ and $t_{2}$ are defined as

$$
\begin{aligned}
& t_{1}=\left(k_{1}-p_{1}\right)^{2}=\left(k_{2}-p_{1}\right)^{2}=u_{1}, \\
& t_{2}=\left(k_{1}-p_{2}\right)^{2}=\left(k_{2}-p_{2}\right)^{2}=u_{2} .
\end{aligned}
$$

Note that $k_{1}=k_{2}$ in the nonrelativistic limit $v \rightarrow 0$. In evaluating the matrix elements, we neglect the lepton masses compared with $m_{W}$ and $m_{\chi}$, while we take into account 
the lepton masses in slepton mass matrices to keep potentially large left-right mixings for the sleptons. ${ }^{4}$ The Gordon decomposition for the s-wave limit, $m_{\chi} \overline{v_{\chi}} \gamma^{\alpha} \gamma_{5} u_{\chi}=$ $-k_{1}^{\alpha} \overline{v_{\chi}} \gamma_{5} u_{\chi}$, can be used to further simplify Eqs. (8)-(10).

The differential cross section for the process $\chi \chi \rightarrow W^{+} \ell \bar{\nu}_{\ell}$ is given by

$$
\frac{d^{2}(\sigma v)_{W \ell \bar{\nu}}}{d E_{W} d E_{\nu}}=\frac{1}{512 \pi^{3} m_{\chi}^{2}} \sum_{\text {spins }}\left|\mathcal{M}_{\mathrm{A}}+\mathcal{M}_{\mathrm{B}}+\mathcal{M}_{\mathrm{C}}\right|^{2}
$$

where $E_{W}$ and $E_{\nu}$ are the energy of the $W$ boson and the neutrino at the center of mass frame. To include the charge-conjugated process as in Eq. (2), the differential cross section in Eq. (12) must be doubled. The helicity sum of the matrix element squared for diagrams A, B and C are given as follows:

$$
\begin{aligned}
\sum_{\text {spins }}\left|\mathcal{M}_{\mathrm{A}}\right|^{2}= & \frac{g^{2}}{4 m_{W}^{2}} \frac{1}{\left(t_{2}-m_{\tilde{\nu}}^{2}\right)^{2}}\left(C_{L}^{(\nu)}\right)^{4} F_{1} \\
\sum_{\text {spins }}\left|\mathcal{M}_{\mathrm{B}}\right|^{2}= & \sum_{I, J} \frac{g^{2}}{4 m_{W}^{2}} \frac{1}{t_{1}-m_{\tilde{\ell} I}^{2}} \frac{1}{t_{1}-m_{\tilde{\ell} J}^{2}} \\
& \times C_{L I}^{(\ell)} C_{L J}^{(\ell)}\left[C_{L I}^{(\ell)} C_{L J}^{(\ell)} F_{1}+\frac{\left.4 m_{\chi}^{2} C_{R I}^{(\ell)} C_{R J}^{(\ell)} F_{2}\right]}{z^{2}}\right. \\
\sum_{\text {spins }}\left|\mathcal{M}_{\mathrm{C}}\right|^{2}= & \sum_{I, J} \frac{g^{2}}{16 m_{W}^{2}} \frac{1}{\left(t_{2}-m_{\tilde{\nu}}^{2}\right)^{2}} \frac{1}{t_{1}-m_{\tilde{\ell} I}^{2}} \frac{1}{t_{1}-m_{\tilde{\ell} J}^{2}}\left(V_{\tilde{\ell}}\right)_{I 1}\left(V_{\tilde{\ell}}\right)_{J 1}\left(C_{L}^{(\nu)}\right)^{2} \\
& \times\left[(x-z)^{2}+4 m_{W}^{2} y\right]\left[C_{L I}^{(\ell)} C_{L J}^{(\ell)} F_{3}+C_{R I}^{(\ell)} C_{R J}^{(\ell)} \cdot 4 m_{\chi}^{2} y\right] \\
\sum_{\text {spins }} \mathcal{M}_{\mathrm{A}} \mathcal{M}_{\mathrm{B}}^{*}+\text { h.c. }= & -\sum_{I} \frac{g^{2}}{2 m_{W}^{2}} \frac{1}{t_{2}-m_{\tilde{\nu}}^{2}} \frac{1}{t_{1}-m_{\tilde{\ell} I}^{2}}\left(C_{L}^{(\nu)}\right)^{2}\left(C_{L I}^{(\ell)}\right)^{2} F_{1}, \\
\sum_{\text {spins }} \mathcal{M}_{\mathrm{A}} \mathcal{M}_{\mathrm{C}}^{*}+\text { h.c. }= & \sum_{I} \frac{g^{2}}{4 m_{W}^{2}} \frac{1}{\left(t_{2}-m_{\tilde{\nu}}^{2}\right)^{2}} \frac{1}{t_{1}-m_{\tilde{\ell} I}^{2}}\left(V_{\tilde{\ell}}\right)_{I 1}\left(C_{L}^{(\nu)}\right)^{3} C_{L I}^{(\ell)} F_{4},
\end{aligned}
$$

\footnotetext{
${ }^{4}$ In the case of electromagnetic bremsstrahlung $\chi \chi \rightarrow \ell^{+} \ell^{-} \gamma$, this leads to an infrared divergence in the total cross section, which should be cancelled by virtual one-loop corrections to the two-body annihilation rate. In the case of $W$-strahlung, however, the total cross section is free from an infrared divergence even when the lepton mass is neglected, since the denominator of the lepton propagators in Eqs. (8) and (9) does not vanish due to $q_{i}^{2} \geq m_{W}^{2}(i=1,2)$.
} 


$$
\begin{aligned}
\sum_{\text {spins }} \mathcal{M}_{\mathrm{B}} \mathcal{M}_{\mathrm{C}}^{*}+\text { h.c. }=-\sum_{I, J} \frac{g^{2}}{4 m_{W}^{2}} \frac{1}{t_{2}-m_{\tilde{\nu}}^{2}} \frac{1}{t_{1}-m_{\tilde{\ell} I}^{2}} \frac{1}{t_{1}-m_{\tilde{\ell} J}^{2}}\left(V_{\tilde{\ell}}\right)_{I 1} C_{L}^{(\nu)} C_{L J}^{(\ell)} \\
\times\left[C_{L I}^{(\ell)} C_{L J}^{(\ell)} F_{4}+\frac{4 m_{\chi}^{2}}{z} C_{R I}^{(\ell)} C_{R J}^{(\ell)} F_{5}\right] .
\end{aligned}
$$

The auxiliary functions $F_{1}, F_{2}, \cdots, F_{5}$ are given by

$$
\begin{aligned}
& F_{1}=x z+2 m_{W}^{2}\left(y-2 m_{\chi}^{2}\right), \\
& F_{2}=z\left(y-2 m_{W}^{2}\right)\left(z-m_{W}^{2}\right)-m_{W}^{2}\left[z\left(y-8 m_{\chi}^{2}\right)+8 m_{W}^{2} m_{\chi}^{2}\right], \\
& F_{3}=x F_{1}-2 m_{W}^{2} y, \\
& F_{4}=(x-z) F_{3}, \\
& F_{5}=y\left[z(x-z)+2 m_{W}^{2}\left(z-4 m_{\chi}^{2}\right)\right],
\end{aligned}
$$

where the Lorentz invariants $x, y$ and $z$ are defined by

$$
x=\left(p_{1}+p_{3}\right)^{2}, \quad y=\left(p_{1}+p_{2}\right)^{2}, \quad z=\left(p_{2}+p_{3}\right)^{2} .
$$

Note that $x+y+z=4 m_{\chi}^{2}+m_{W}^{2}$, where $m_{\chi}$ and $m_{W}$ denote the mass of the neutralino and $W$ boson, respectively. The Lorentz invariants in Eqs. (11) and (20) can be written in terms of the energies as $x=4 m_{\chi}\left(m_{\chi}-E_{\nu}\right), y=4 m_{\chi}\left(m_{\chi}-E_{W}\right)+$ $m_{W}^{2}, z=4 m_{\chi}\left(m_{\chi}-E_{\ell}\right), t_{1}=\frac{z}{2}-m_{\chi}^{2}, t_{2}=\frac{x}{2}-m_{\chi}^{2}$, where $E_{\ell}$ is the energy of the charged lepton at the center of mass frame of the initial neutralinos, and the lepton mass is neglected.

Let us discuss the behavior in the heavy slepton limit: $m_{\tilde{\ell} I}=m_{\tilde{\nu}} \equiv \tilde{m} \rightarrow \infty$. The slepton mass dependence of each amplitude is $\mathcal{M}_{\mathrm{A}}, \mathcal{M}_{\mathrm{B}} \sim 1 / \tilde{m}^{2}$, and $\mathcal{M}_{\mathrm{C}} \sim$ $1 / \tilde{m}^{4}$. However, by summing up Eqs. (13), (14) and (16), the leading $1 / \tilde{m}^{2}$ terms cancel out between diagrams A and B, resulting in the total amplitude suppressed as $\sim 1 / \tilde{m}^{4}[11]$. On the other hand, the amplitude for the leptonic two-body process, $\chi \chi \rightarrow \tau^{+} \tau^{-}$, is suppressed only by $\sim 1 / \tilde{m}^{2}$. Therefore, the ratio $(\sigma v)_{W \tau \nu} /(\sigma v)_{\tau^{+} \tau^{-}}$ falls down for heavy sleptons.

We also evaluate neutrino spectra at injection from the center of the Sun. The primary neutrino spectrum via $\chi \chi \rightarrow W \ell \bar{\nu}$ is obtained by integrating Eq. (12) over $E_{W}$ as

$$
\frac{d(\sigma v)_{W \ell \bar{\nu}}}{d E_{\nu}}=\int_{E_{W}^{\min }\left(E_{\nu}\right)}^{E_{W}^{\max }} d E_{W} \frac{d^{2}(\sigma v)_{W \ell \bar{\nu}}}{d E_{W} d E_{\nu}}
$$


where

$$
\begin{aligned}
E_{W}^{\min }\left(E_{\nu}\right) & =m_{\chi}-E_{\nu}+\frac{m_{W}^{2}}{4\left(m_{\chi}-E_{\nu}\right)} \\
E_{W}^{\max } & =m_{\chi}\left(1+\frac{m_{W}^{2}}{4 m_{\chi}^{2}}\right) .
\end{aligned}
$$

The cross section is obtained by integrating Eq. (21) over $E_{\nu}$ in the range $0<E_{\nu}<$ $m_{\chi}\left(1-\frac{m_{W}^{2}}{4 m_{\chi}^{2}}\right)$. This integration can be done analytically, although the expressions are lengthy.

The secondary neutrino spectra from decay of the $W$ boson and the tau lepton are evaluated as follows. The neutrino spectrum via $W$-boson decay is written as

$$
\left.\frac{d(\sigma v)_{W \ell \bar{\nu}}}{d E_{\nu}}\right|_{\text {from } W}=\int_{m_{W}}^{E_{W}^{\max }} d E_{W} \frac{d(\sigma v)_{W \ell \bar{\nu}}}{d E_{W}}\left[\left(\frac{d N_{\nu}}{d E_{\nu}}\right)_{W}\left(E_{W}, E_{\nu}\right)\right],
$$

where $\left(\frac{d N_{\nu}}{d E_{\nu}}\right)_{W}\left(E_{W}, E_{\nu}\right)$ is the neutrino energy distribution per $W$-boson decay with energy $E_{W}$, and $d(\sigma v)_{W \ell \bar{\nu}} / d E_{W}$ is the $W$-boson spectrum obtained by integrating Eq. (12) over $E_{\nu}$. The secondary neutrino spectrum from tau decay is obtained in a similar fashion using the neutrino distribution per tau decay, $\left(\frac{d N_{\nu}}{d E_{\nu}}\right)_{\tau}\left(E_{\tau}, E_{\nu}\right)$. In evaluating the contributions via relevant two-body processes $\chi \chi \rightarrow \tau^{+} \tau^{-}, t \bar{t}, b \bar{b}$ and $W^{+} W^{-}$, we further need the neutrino distributions from the top and bottom quarks. Neutrino distribution $\left(\frac{d N_{\nu}}{d E_{\nu}}\right)_{i}\left(E_{i}, E_{\nu}\right)$ from the parent particle $(i=W, \tau, t, b)$ with energy $E_{i}$ is affected by matter effects in the Sun. We neglect decay of the light quarks and the muon, since they stop before decay in the Sun. We also neglect the contribution of the charm quark, since it is subdominant compared with that of the bottom quark. For the energy distributions $\left(\frac{d N_{\nu}}{d E_{\nu}}\right)_{i}$ in the Sun, we use the result of Ref. [24] where the distributions are obtained with the Monte Carlo code PYTHIA [25]. For the energy of the parent particles not tabulated in the reference, we simply adopt linear interpolations.

\section{NARROW WIDTH APPROXIMATION FOR $\chi \chi \rightarrow W t b$}

In this section, we discuss the relation between the massive weak bremsstrahlung process $\chi \chi \rightarrow W t b$ and the corresponding two-body process $\chi \chi \rightarrow t \bar{t}$ using a narrow width approximation. 
The cross section for $W t b$ can be obtained from $\mathcal{M}_{\mathrm{A}}, \mathcal{M}_{\mathrm{B}}$ and $\mathcal{M}_{\mathrm{C}}$ by replacing $\nu$ and $\ell$ with $t$ and $b$, respectively, and taking both left- and right-handed stops into account. When $m_{\chi}>m_{t}$, the top quark pair production $\chi \chi \rightarrow t \bar{t}$ opens up. Then, the three-body cross section for $\chi \chi \rightarrow W t b$ calculated with only $\mathcal{M}_{\mathrm{A}}$ reduces to the cross section of the two-body process $\chi \chi \rightarrow t \bar{t}$ evaluated with only $t$ - and $u$-channel diagrams. In this sense, the massive weak bremsstrahlung $\chi \chi \rightarrow W^{+} \bar{t} b$ is included in the two-body process $\chi \chi \rightarrow t \bar{t}$ followed by the on-shell top quark decay for $m_{\chi}$ $>m_{t}[20]$. On the other hand, the leptonic $W$-strahlung in Eq. (2) can never be included in any two-body process, since a $W$-boson emission from on-shell lepton is kinematically forbidden.

We have calculated the cross section for the $W t b$ final state in the same way as the leptonic case. Integrating the differential cross section $d^{2}(\sigma v)_{W \bar{t} b}^{(\tilde{t})} /\left(d E_{W} d E_{t}\right)$ over $E_{W}$, the top quark energy distribution via only stop exchange can be obtained as

$$
\begin{aligned}
\frac{d(\sigma v)_{W \bar{t} b}^{(\tilde{t})}}{d E_{t}}=\sum_{I, J} & \frac{N_{c} g^{2}}{512 \pi^{3} m_{W}^{2}} \sqrt{E_{t}^{2}-m_{t}^{2}} \frac{\Delta_{t}+m_{t}^{2}+2 m_{W}^{2}}{\Delta_{t}^{2}+\Gamma_{t}^{2} m_{t}^{2}} \\
& \times \frac{\left(\Delta_{t}+m_{t}^{2}-m_{W}^{2}\right)^{2}}{\Delta_{t}+m_{t}^{2}} \frac{1}{t_{2}-m_{\tilde{t} I}^{2}} \frac{1}{t_{2}-m_{\tilde{t} J}^{2}} \\
& \times\left[\left(C_{L I}^{(t)}\right)^{2}\left(C_{L J}^{(t)}\right)^{2} f_{t}\left(E_{t}\right)+m_{t} m_{\chi}\left(\left(C_{L I}^{(t)}\right)^{2} C_{R J}^{(t)} D_{L J}^{(t)}+\left(C_{L J}^{(t)}\right)^{2} C_{R I}^{(t)} D_{L I}^{(t)}\right)\right. \\
& \left.\quad+\frac{m_{\chi}^{2} f_{t}\left(E_{t}\right)}{\Delta_{t}+m_{t}^{2}} C_{R I}^{(t)} D_{L I}^{(t)} C_{R J}^{(t)} D_{L J}^{(t)}\right], \quad(24)
\end{aligned}
$$

where $\Delta_{t}=x-m_{t}^{2}=4 m_{\chi}\left(m_{\chi}-E_{t}\right), \Gamma_{t}$ is the decay width for the top quark, $N_{c}=$ 3 is the color factor, and

$$
f_{t}\left(E_{t}\right)=2 E_{t}\left(m_{\chi}-E_{t}\right)+m_{t}^{2} .
$$

The coupling constants $C_{L I}^{(t)}$ and $C_{R I}^{(t)}$ can be defined in a similar fashion as $C_{L I}^{(\ell)}$ and $C_{R I}^{(\ell)}$ in Eq. (4). The constant $D_{L I}^{(t)}$ is defined by

$$
m_{\chi} D_{L I}^{(t)}=2 m_{\chi} C_{L I}^{(t)}+m_{t} C_{R I}^{(t)}
$$

Integration of Eq. (24) over $E_{t}$ gives the cross section $(\sigma v)_{W \bar{t} b}^{(\tilde{t})}$. If the integration range includes the pole $E_{t}=m_{\chi}\left(x=m_{t}^{2}\right)$, the narrow width approximation can be justified where the propagator of the top quark can be replaced with the delta function as

$$
\frac{1}{\left(x-m_{t}^{2}\right)^{2}+\Gamma_{t}^{2} m_{t}^{2}} \approx \frac{\pi}{\Gamma_{t} m_{t}} \delta\left(x-m_{t}^{2}\right)
$$


Under this approximation, the integration of Eq. (24) over $x$ (equivalently over $E_{t}$ ) indeed reduces to the two-body s-wave cross section via $t$ - and $u$-channel stop exchange [3] (see Appendix B)

$$
(\sigma v)_{t \bar{t}}^{(\tilde{t})}=\frac{N_{c}}{32 \pi} \sqrt{1-\frac{m_{t}^{2}}{m_{\chi}^{2}}}\left|\sum_{I=1,2} \frac{m_{t}\left(\left(C_{L I}^{(t)}\right)^{2}+\left(C_{R I}^{(t)}\right)^{2}\right)+2 m_{\chi} C_{L I}^{(t)} C_{R I}^{(t)}}{m_{t}^{2}-m_{\chi}^{2}-m_{\tilde{t} I}^{2}}\right|^{2},
$$

where we use the expression for the top-quark decay width [26]

$$
\Gamma_{t}=\frac{g^{2}}{64 \pi} \frac{m_{t}^{3}}{m_{W}^{2}}\left(1-\frac{m_{W}^{2}}{m_{t}^{2}}\right)^{2}\left(1+2 \frac{m_{W}^{2}}{m_{t}^{2}}\right) .
$$

We neglect the s-channel contributions for the three-body processes for simplicity. This is a good approximation for the leptonic process $\chi \chi \rightarrow W \ell \nu$. For the $W t b$ final state, however, this is not a good approximation, since squarks are extremely heavy in the present analysis. In our numerical calculation, we use the total two-body expression for $\chi \chi \rightarrow t \bar{t}$ in Eq. (B1) rather than in Eq. (28). Therefore, our result for the $W t b$ final state below the $t \bar{t}$ threshold $m_{\chi} \lesssim m_{t}$ is not smoothly connected to that for the $t \bar{t}$ final state. This does not affect our conclusion, since the $W t b$ contribution below the threshold is subdominant in the parameter range we consider in the present analysis.

\section{NUMERICAL RESULTS}

In this section, we present our numerical results. In this analysis, we examine the phenomenological MSSM, where various SUSY parameters are chosen freely. In order to find the parameter ranges where the weak bremsstrahlung is important, we consider the scenario that the sleptons lie below the TeV scale, while the squarks are extremely heavy. Throughout the analyses, squark mass parameters are taken as $m_{\tilde{q}}$ $\gtrsim 10 \mathrm{TeV}$ for all the squarks to be consistent with the null results of superparticle searches at the LHC [27]. The pseudoscalar Higgs boson mass is fixed at $m_{A}=2 \mathrm{TeV}$ in every figure. For the slepton mass parameters, we assume a common value $m_{\tilde{e}}=$ $m_{\tilde{\mu}}$ for the left- and right-handed soft SUSY breaking masses for the selectrons and smuons, while the left- and right-handed stau mass parameters, $m_{\tilde{\tau} L}$ and $m_{\tilde{\tau} R}$, are chosen independently. For the trilinear scalar couplings, we vary $A_{t}, A_{b}$ and $A_{\tau}$ for the stop, sbottom and stau, respectively. All the others are set to zero: $A_{q}=0(q \neq$ $t, b)$ for the other squarks, and $A_{e}=A_{\mu}=0$. 
The s-wave cross section times the relative velocity, $\sigma v$, for the weak bremsstrahlung processes $\chi \chi \rightarrow W \ell \nu$, including both $W^{+} \ell^{-} \bar{\nu}_{\ell}$ and $W^{-} \ell^{+} \nu_{\ell}$, are plotted in Fig. 2 as a function of $m_{\chi}$ together with the contributions of the relevant two-body processes. In Fig. 2 (a), the MSSM parameters are chosen as $\tan \beta=2, \mu$ $=1 \mathrm{TeV}, m_{\tilde{q}}=14 \mathrm{TeV}$, and $m_{\tilde{e}}=m_{\tilde{\mu}}=m_{\tilde{\tau} L}=m_{\tilde{\tau} R}=240 \mathrm{GeV}$. The trilinear coupling $A_{t}$ is chosen to satisfy the Higgs mass constraint $m_{h} \sim 125 \mathrm{GeV}[28,29]$, although the trilinear coupling for the squarks are irrelevant in the present analysis where squark exchange diagrams are sufficiently suppressed by the heavy squark masses. The bold solid line corresponds to the sum of all the leptonic processes, $\sum_{\ell} W \ell \bar{\nu}_{\ell}+$ h.c. The contributions of $W e \nu_{e}$ and $W \mu \nu_{\mu}$ are identical, $(\sigma v)_{W e \nu_{e}}=(\sigma v)_{W \mu \nu_{\mu}}$, which are essentially described by the simplified expression in Appendix A. On the other hand, the result for $W \tau \nu_{\tau}$ is slightly larger $(\sigma v)_{W \tau \nu_{\tau}} \sim 1.2 \times(\sigma v)_{W e \nu_{e}}$, where the difference originates from the left-right mixing for the staus. The contributions of the relevant two-body processes are shown with thin lines. ${ }^{5}$ The solid, dashed, dot-dashed and dotted lines correspond to $\tau^{+} \tau^{-}, t \bar{t}, b \bar{b}$ and $W^{+} W^{-}$, respectively. For a relatively large value of $m_{\chi} \lesssim m_{\tilde{\ell}}$, the weak bremsstrahlung dominates over the two-body contributions. The range of $m_{\chi}$ filled with gray corresponds to the cosmologically allowed region where the relic abundance constraint $0.11<\Omega_{\chi} h^{2}<0.13$ is satisfied. The relic abundance is obtained using DarkSUSY [30]. In the present scenario with a bino-like LSP, the relic density is typically too large. The allowed region in Fig. 2 (a) appears with the help of slepton coannihilations which lead to an effective enhancement of the pair annihilation cross section for $m_{\chi} \approx m_{\tilde{\ell}}[31-35]$.

In Fig. 2 (b), the soft mass parameter for the right-handed stau is taken to be larger than the other slepton mass parameters as $m_{\tilde{\tau} R}=480 \mathrm{GeV}$. In this case, the contributions of $W e \nu_{e}$ and $W \mu \nu_{\mu}$ are the same as in Fig. 2 (a), while that of $\tau^{+} \tau^{-}$gets suppressed due to the larger $m_{\tilde{\tau} R}$. The contribution of $W \tau \nu_{\tau}$ becomes identical to those of $W e \nu_{e}$ and $W \mu \nu_{\mu}$, since the effect of left-right mixing for the staus, $\sim m_{\tau}\left(A_{\tau}-\right.$ $\mu \tan \beta) /\left(m_{\tilde{\tau} L}^{2}-m_{\tilde{\tau} R}^{2}+\delta_{\tau}\right)$, is reduced by taking different values for $m_{\tilde{\tau} R}$ and $m_{\tilde{\tau} L}$, where $\delta_{\tau} \sim-0.04 m_{Z}^{2} \cos 2 \beta$. Thus the relative magnitude of weak bremsstrahlung is increased compared with the result in Fig. 2 (a). The weak bremsstrahlung is dominant for all values of $m_{\chi}$.

In Fig. 2 (c), the soft mass parameters for both the left- and right-handed stau are

\footnotetext{
${ }^{5}$ The p-wave contributions $(\sigma v)_{2}^{\mathrm{p} \text {-wave }} \sim v^{2} g^{44} /\left(192 \pi m_{\chi}^{2}\right)$ are typically smaller than the relevant
} s-wave contributions for the relative velocity $v \sim 10^{-3}$ in the galactic halo. 
taken to be larger than that for the selectron and smuon as $m_{\tilde{\tau} L}=m_{\tilde{\tau} R}=480 \mathrm{GeV}$. The bold dashed line represents the result for only $W \tau \nu_{\tau}$. The contribution of $W \tau \nu_{\tau}$ is reduced due to the larger $m_{\tilde{\tau} L}$.

Figure $2(\mathrm{~d})$ is the result for $\tan \beta=10, \mu=3 \mathrm{TeV}$, and $m_{\tilde{q}}=12 \mathrm{TeV}$, where the mass parameters for staus are taken to be larger as $m_{\tilde{\tau} L}=m_{\tilde{\tau} R}=2 \mathrm{TeV}$. For a large $\tan \beta$, the contribution of the $\tau^{+} \tau^{-}$final state is enhanced due to the larger left-right mixing, while $W e \nu_{e}$ and $W \mu \nu_{\mu}$ remain unchanged. However, by taking much larger masses for staus than in Figs. 2 (a)-(c), the weak bremsstrahlung can be dominant over the two-body processes. The process $W \tau \nu_{\tau}$ is suppressed as $\sim 1 / m_{\tilde{\tau} L}^{8}$. The bold dotted line represents the contribution of the $W t b$ final state below the $t \bar{t}$ threshold $m_{\chi}<m_{t}$. This proceeds via off-shell top quark effect $\chi \chi \rightarrow t^{*} \bar{t}$ followed by the decay $t^{*} \rightarrow W b[20]$. In the present analysis, we include only $t$ - and $u$-channel diagrams for the $W t b$ final state as explained in Sec. IV. If we had included the $s$-channel diagrams for $W t b$, the result below the threshold would be smoothly connected to the two-body $t \bar{t}$ result.

We define the ratio $R=(\sigma v)_{3 W} /(\sigma v)_{2}$, where $(\sigma v)_{3 W}$ is the total leptonic $W$ strahlung contribution, and $(\sigma v)_{2}=\sum_{f=t, b, \tau}(\sigma v)_{f \bar{f}}+(\sigma v)_{W W}$ is the sum of all the relevant two-body contributions included in the present analysis. ${ }^{6}$ The contours of the ratio $R$ are plotted in the $\left(\mu, M_{2}\right)$ plane in Fig. 3 with bold lines. The relavant parameters for Figs. 3 (a)-(d) are taken to be the same as in Figs. 2 (a)-(d), respectively. The contours of $(\sigma v)_{3 W}$ are shown with thin lines. As $M_{2}$ gets across $2 m_{t} \sim 350 \mathrm{GeV}$, the ratio becomes small suddenly due to opening of the $t \bar{t}$ channel. The strip filled with gray corresponds to the cosmologically allowed region with the correct $\Omega_{\chi} h^{2}$. In Fig. 3 (a), the $W$-strahlung cross section $(\sigma v)_{3 W}$ becomes larger as $M_{2}$ increases. In the cosmologically allowed range, the weak bremsstrahlung can be comparable with the two-body processes for $800 \mathrm{GeV} \lesssim \mu \lesssim 1100 \mathrm{GeV}$. The area filled with light gray is excluded where the lighter stau is lighter than the neutralino. The figure includes a small $\mu$ range where the LSP is higgsino-like. In this region, the $W W$ final state becomes dominant, though cosmologically allowed regions exist for higgsino-like LSP. Therefore, the weak bremsstrahlung is negligible for a higgsino-like LSP in the present analysis. We do not show the result for $\mu<0$, since the behavior is similar to that for $\mu>0$.

\footnotetext{
${ }^{6}$ We have not inluded $Z Z, H V$ and $H H$ final states in the present analysis for simplicity, where $V$ $=W / Z$, and $H$ represents one of the Higgs bosons.
} 
In Figs. 3 (b) and (c), where the mass parameters for staus are larger, the contribution of $W \ell \nu_{\ell}$ is similar to that in Fig. 3 (a). However, the ratio can be significantly larger than in Fig. 3 (a), since the relevant two-body final state, $\tau^{+} \tau^{-}$, gets smaller than in Fig. 3 (a). In Fig. 3 (d), the ratio is further enhanced by taking a large value for stau mass parameters as $m_{\tilde{\tau} L}=m_{\tilde{\tau} R}=2 \mathrm{TeV}$ for $\tan \beta=10$.

Finally, neutrino spectra at injection in the Sun are shown in Fig. 4. The panel (a) is the result for $\tan \beta=2, M_{2}=450 \mathrm{GeV}, \mu=1 \mathrm{TeV}, m_{\tilde{q}}=14 \mathrm{TeV}$, and $m_{\tilde{e}}$ $=m_{\tilde{\mu}}=m_{\tilde{\tau} L}=m_{\tilde{\tau} R}=240 \mathrm{GeV}$. The bold solid line corresponds to the primary neutrino spectrum of weak bremsstrahlung including all the flavors and the charge conjugated states. The bold dashed and dotted lines are the results for the secondary neutrinos from the tau lepton and the $W$ boson, respectively, produced via weak bremsstrahlung. The contributions of two-body processes are drawn with thin lines. The neutralino mass in this case is $m_{\chi} \approx 221.9 \mathrm{GeV}$. One can see that the primary neutrino from weak bremsstrahlung can give a significant contribution particularly in the high energy range $0.8 m_{\chi} \lesssim E_{\nu} \lesssim m_{\chi}$. The result for $m_{\tilde{\tau} L}=m_{\tilde{\tau} R}=480 \mathrm{GeV}$ is shown in the panel (b). In this case, the weak bremsstrahlung is dominant for the wide range of $E_{\nu}$, since the $\tau^{+} \tau^{-}$final state is suppressed by the large $m_{\tilde{\tau} L}$ and $m_{\tilde{\tau} R}$. It is notable that primary neutrino contributions of weak bremsstrahlung are nearly flavor independent for a common slepton mass, while the largest contribution among the two-body process, $\tau^{+} \tau^{-}$, produces mainly tau neutrinos in the Sun. Hence $W \ell \nu$ can strongly affect the flavor contents of energetic neutrinos [14].

Even when the three-body final state $W \ell \nu$ is not the dominant channel in the total cross section, the neutrino spectrum at high energies, $E_{\nu} \lesssim m_{\chi}$, can still be dominated by $W \ell \nu$. The energetic neutrinos mainly originate from the internal bremsstrahlung, diagram $\mathrm{C}$ in Fig. 1. Indeed, in Fig. 4(a), the total cross section of the three-body process is slightly smaller than the sum of the two-body results. Nevertheless, the three-body process is dominant in the high energy region in Fig. 4(a).

It must be kept in mind that one should take into account the usual bremsstrahlung effect $(\sigma v)_{3 \gamma}$ as well to evaluate the total three-body cross section. However, as far as a neutrino flux is concerned, the weak bremsstrahlung is expected to give the major contribution. Also, $Z$-boson strahlung processes, e.g., $\chi \chi \rightarrow Z \ell^{+} \ell^{-}$, should be included to discuss the total contributions from weak bremsstrahlung. 


\section{CONCLUSIONS}

We have examined the effects of electroweak bremsstrahlung on the bino-like neutralino dark matter pair annihilations in the MSSM. We have calculated the nonrelativistic pair annihilation cross sections and neutrino spectra via $W$-strahlung, $\chi \chi \rightarrow$ $W \ell \bar{\nu}$. It has been shown that the electroweak bremsstrahlung can give a dominant contribution to the cross section for some parameter regions which include cosmologically allowed ranges with the observed relic abundance. It has been found that the weak bremsstrahlung processes can give characteristic signals in the neutrino spectrum at injection in the Sun.

In the present analyses, we considered extremely heavy squarks. When the squark masses are comparable with the slepton masses, the weak bremsstrahlung typically gives only a subdominant contribution for $m_{\chi}>m_{t}$ due to the unsuppressed $t \bar{t}$ final state. Below the $t \bar{t}$ threshold, the $W t b$ final state can be relevant as shown in Ref. [20].

\section{Acknowledgments}

This work was supported in part by a CST grant for Gakujyutsusyo, Nihon University. The authors are very grateful to S. Naka, S. Deguchi and A. Miwa for useful discussions and comments.

\section{Appendix A: Unbroken SU(2) limit in the slepton sector}

In this section, we provide a simple expression for the unbroken $S U(2)_{L}$ limit in the slepton sector, taking the common slepton masses $m_{\tilde{\nu}}=m_{\tilde{\ell} I} \equiv \tilde{m}$, and the common left-handed slepton coupling constants $C_{L}^{(\nu)}=C_{L 1}^{(\ell)} \equiv C_{L}$ with $C_{L 2}^{(\ell)}=C_{R I}^{(\ell)}=0$, and keeping $m_{W} \neq 0$. This corresponds to the choices $N_{12}=0, h_{\ell}=0,\left(\widetilde{V}_{\ell}\right)_{11}=1$ and $\left(\widetilde{V}_{\ell}\right)_{12}=0$ in Eq. (4). In this limit, the neutralino is a pure bino, and only the lefthanded sleptons contribute. Writing the matrix element $\mathcal{M}=\mathcal{M}_{\mathrm{A}}+\mathcal{M}_{\mathrm{B}}+\mathcal{M}_{\mathrm{C}}$ as $\mathcal{M}=\varepsilon_{3}^{\mu} \mathcal{M}_{\mu}$, it is confirmed that the Ward identity $p_{3}^{\mu} \mathcal{M}_{\mu}=0$ is satisfied using $\not_{1} u_{\ell}$ $=\not p_{2} v_{\nu}=0$ [11]. This implies that the longitudinal polarization of the $W$ boson does not contribute to the s-wave amplitude in this limit.

The differential cross section for $\chi \chi \rightarrow W \ell \bar{\nu}$ can be greatly simplified as

$$
\frac{d^{2}(\sigma v)_{W \ell \bar{\nu}}}{d E_{W} d E_{\nu}}=\frac{g^{2} C_{L}^{4}}{4096 \pi^{3} m_{\chi}^{2}} \frac{1}{\left(t_{1}-\tilde{m}^{2}\right)^{2}} \frac{1}{\left(t_{2}-\tilde{m}^{2}\right)^{2}} y\left(x^{2}+z^{2}-8 m_{\chi}^{2} m_{W}^{2}\right) .
$$


Integrating over $d E_{\nu}$ and $d E_{W}$ analytically, Eq. (A1) reduces to the cross section found in Ref. [11]. Note that the cross section is severely suppressed as $\sim 1 / \tilde{m}^{8}$ in the heavy slepton limit.

In the numerical analyses in Sec. V, the $S U(2)_{L}$ breaking effects lead to $m_{\tilde{\nu}}^{2}-m_{\tilde{\ell} 1}^{2}$ $\sim m_{Z}^{2} \cos 2 \beta \cos ^{2} \theta_{W}$ without the left-right mixing for the sleptons. In the presence of the mass splitting $m_{\tilde{\nu}}-m_{\tilde{\ell} 1} \neq 0$, a longitudinal $W$-boson emission $\chi \chi \rightarrow W_{L} \ell \bar{\nu}_{\ell}$ can enhance the cross section [19]. ${ }^{7}$

\section{Appendix B: Two-body processes}

In this appendix, the s-wave cross sections for the relevant two-body processes are summarized for convenience [3].

The s-wave cross section for the fermion pair production $\chi \chi \rightarrow f \bar{f}$ is given by

$$
(\sigma v)_{f \bar{f}}=\frac{N_{c}}{2 \pi} \sqrt{1-\frac{m_{f}^{2}}{m_{\chi}^{2}}}\left|F_{A}+F_{Z}+\sum_{I=1}^{2} F_{\tilde{f} I}\right|^{2},
$$

where

$$
\begin{aligned}
F_{A} & =\frac{C_{P}^{f f A} C_{P}^{\chi \chi A}}{4 m_{\chi}^{2}-m_{A}^{2}+i \Gamma_{A} m_{A}} m_{\chi}, \\
F_{Z} & =\frac{C_{A}^{f f Z} C_{A}^{\chi \chi Z}}{4 m_{\chi}^{2}-m_{Z}^{2}+i \Gamma_{Z} m_{Z}} \frac{m_{f}\left(4 m_{\chi}^{2}-m_{Z}^{2}\right)}{m_{Z}^{2}}, \\
F_{\tilde{f} I} & =\frac{1}{4} \cdot \frac{m_{f}\left[\left(C_{L I}^{(f)}\right)^{2}+\left(C_{R I}^{(f)}\right)^{2}\right]+2 m_{\chi} C_{L I}^{(f)} C_{R I}^{(f)}}{m_{f}^{2}-m_{\chi}^{2}-m_{\tilde{f} I}^{2}} .
\end{aligned}
$$

The quantities $F_{A}, F_{Z}$ and $F_{\tilde{f} I}$ represent the amplitude of $s$-channel pseudoscalar Higgs boson $(A)$ exchange, $s$-channel $Z$-boson exchange, and $t$ - and $u$-channel sfermion exchange, respectively. The constant $N_{c}$ is the color factor: $N_{c}=3$ for quark pairs, and $N_{c}=1$ for leptons. The coupling constants $C_{P}^{f f A}$ and $C_{P}^{\chi \chi A}$ describe the interaction of the pseudoscalar Higgs $A$ with bilinears $\bar{f} i \gamma_{5} f$ and $\bar{\chi} i \gamma_{5} \chi$, respectively. The coupling constants $C_{A}^{f f Z}$ and $C_{A}^{\chi \chi Z}$ determine the axial vector interaction of the $Z$

\footnotetext{
${ }^{7}$ Neglecting the slepton mixing, but still taking the mass splitting into account, our result agrees with the one found in Ref. [19].
} 
boson with bilinears $\bar{f} \gamma_{\mu} \gamma_{5} f$ and $\bar{\chi} \gamma_{\mu} \gamma_{5} \chi$, respectively. The expressions for these coupling constants can be found in Ref. [23]. The decay widths for the $Z$ boson and the pseudoscalar Higgs, $\Gamma_{Z}$ and $\Gamma_{A}$, are taken into account. Note that the contribution of the sfermion exchange, $F_{\tilde{f} I}$, includes a factor of the fermion mass $m_{f}$, since $C_{R I}^{(f)}$ $\left(C_{L I}^{(f)}\right)$ is proportional to $m_{f}$ for $I=1(I=2)$.

The s-wave cross section for the $W$-boson pair production $\chi \chi \rightarrow W^{+} W^{-}$via chargino exchange diagrams is given by

$$
(\sigma v)_{W W}=\frac{1}{2 \pi} \sqrt{1-\frac{m_{W}^{2}}{m_{\chi}^{2}}}\left(m_{\chi}^{2}-m_{W}^{2}\right)\left|\sum_{p=1,2} \frac{\left(C_{V}^{\chi_{p}^{+} \chi W^{-}}\right)^{2}+\left(C_{A}^{\chi_{x}^{+} \chi W^{-}}\right)^{2}}{m_{\chi}^{2}+m_{\chi_{p}^{+}}^{2}-m_{W}^{2}}\right|^{2},
$$

where $m_{\chi_{p}^{+}}$denotes the chargino mass $(p=1,2)$. The coupling constants $C_{V}^{\chi_{p}^{+} \chi W^{-}}$and $C_{A}^{\chi_{A}^{+} \chi W^{-}}$defined in Ref. [23] describe the neutralino-chargino-W vector/axial-vector interactions.

Neutrino spectra for the two-body processes can be obtained from Eq. (23) by replacing the primary spectrum with the delta function distribution. For instance, the neutrino spectrum via $\chi \chi \rightarrow W^{+} W^{-}$can be written as

$$
\left.\frac{d(\sigma v)_{W W}}{d E_{\nu}}\right|_{\text {from } W}=(\sigma v)_{W W}\left[\left(\frac{d N_{\nu}}{d E_{\nu}}\right)_{W}\left(m_{\chi}, E_{\nu}\right)\right]
$$

by replacing $d(\sigma v)_{W \ell \bar{\nu}} / d E_{W}$ in Eq. (23) with $(\sigma v)_{W W} \delta\left(E_{W}-m_{\chi}\right)$.

[1] E. Kolb and M. Turner, The Early Universe, Addison-Wesley (1990).

[2] P.A.R. Ade et al., [Planck Collaboration], arXiv:1303.5076.

[3] For reviews on supersymmetric dark matter, see for instance, G. Jungman, M. Kamionkowski and K. Griest, Phys. Rep. 267, 195 (1996).

[4] For recent comprehensive reviews on particle dark matter, see for instance, G. Bertone (ed.), "Particle dark matter: Observations, models and searches," Cambridge U.P., UK (2010).

[5] For a review on the MSSM, see for instance, H.P. Nilles, Phys. Rep. 110, 1 (1984).

[6] H.E. Haber and G.L. Kane, Phys. Rep. 117, 75 (1985); J.F. Gunion and H.E. Haber, Nucl. Phys. B 272, 1 (1986).

[7] H. Goldberg, Phys. Rev. Lett. 50, 1419 (1983); J.R. Ellis, J.S. Hagelin, D.V. Nanopoulos, K.A. Olive and M. Srednicki, Nucl. Phys. B 238, 453 (1984). 
[8] L. Bergstrom, Phys. Lett. B 225, 372 (1989); R. Flores, K.A. Olive and S. Rudaz, Phys. Lett. B 232, 377 (1989).

[9] T. Bringmann, L. Bergstrom and J. Edsjo, J. High Energy Phys. 0801, 049 (2008) [arXiv:0710.3169]; L. Bergstrom, T. Bringmann and J. Edsjo, Phys. Rev. D 78, 103520 (2008) [arXiv:0808.3725].

[10] N.F. Bell, J.B. Dent, T.D. Jacques, L.M. Krauss and T.J. Weiler, Phys. Rev. D 83, 013001 (2011) [arXiv:1009.2584].

[11] N.F. Bell, J.B. Dent, A.J. Galea, T.D. Jacques, L.M. Krauss and T.J. Weiler, Phys. Lett. B 706, 6 (2011) [arXiv:1104.3823].

[12] V. Barger, Y. Gao, W.Y. Keung and D. Marfatia, Phys. Rev. D 80, 063537 (2009) [arXiv:0906.3009]; Barger, W. -Y. Keung and D. Marfatia, Phys. Lett. B 707, 385 (2012) [arXiv:1111.4523].

[13] N.F. Bell, J.B. Dent, T.D. Jacques and T.J. Weiler, Phys. Rev. D 84, 103517 (2011) [arXiv:1101.3357].

[14] N.F. Bell, A.J. Brennan and T.D. Jacques, J. Cosmo. Astropart. Phys. 1210, 045 (2012) [arXiv:1206.2977].

[15] K. Fukushima, Y. Gao, J. Kumar and D. Marfatia, Phys. Rev. D 86, 076014 (2012) [arXiv:1208.1010].

[16] P. Ciafaloni, M. Cirelli, D. Comelli, A.De Simone, A. Riotto and A. Urbano, J. Cosmo. Astropart. Phys. 1110, 034 (2011) [arXiv:1107.4453]; P. Ciafaloni, D. Comelli, A. De Simone, A. Riotto and A. Urbano, J. Cosmo. Astropart. Phys. 1206, 016 (2012) [arXiv:1202.0692].

[17] J. Kumar and P. Sandick. arXiv:1303.2384.

[18] N. Baro, M. Beneke, M. Kramer, and M. Rummel, PoS IDM2010, 059 (2011).

[19] M. Garny, A. Ibarra and S. Vogl, J. Cosmo. Astropart. Phys. 1107, 028 (2011) [arXiv:1105.5367]; M. Garny, A. Ibarra and S. Vogl, J. Cosmo. Astropart. Phys. 1204, 033 (2012) [arXiv:1112.5155].

[20] X. Chen and M. Kamionkowski, J. High Energy Phys. 9807, 001 (1998) [hep$\mathrm{ph} / 9805383]$.

[21] P. Ciafaloni, D. Comelli, A. De Simone, E. Morgante, A. Riotto and A. Urbano, arXiv:1305.6391.

[22] P. Ciafaloni, M. Cirelli, D. Comelli, A. De Simone, A. Riotto and A. Urbano, J. Cosmo. Astropart. Phys. 1106, 018 (2011) [arXiv:1104.2996]; M. Kachelriess, P.D. Serpico and M.Aa. Solberg, Phys. Rev. D 80, 123533 (2009) [arXiv:0911.0001]; J. Kearney and A. Pierce, Phys. Rev. D 86, 043527 (2012) [arXiv:1202.0284]. 
[23] T. Nihei, L. Roszkowski and R. Ruiz de Austri, J. High Energy Phys. 0203, 031 (2002).

[24] M. Cirelli, N. Fornengo, T. Montaruli, I.A. Sokalski, A. Strumia and F. Vissani, Nucl. Phys. B 727, 99 (2005), Erratum-ibid. B 790 (2008) 338.

[25] T. Sjöstrand et.al., Comput. Phys. Commun 135, 238-259 (2001).

[26] J. Beringer et al. [Particle Data Group], Phys. Rev. D 86, 010001 (2012).

[27] ATLAS Collaboration, Phys. Lett. B 710, 67 (2012) [arXiv:1109.6572], CMS Collaboration, Phys. Rev. Lett. 107, 221804 (2011) [arXiv:1109.2352].

[28] ATLAS Collaboration, G. Aad et al., Phys. Lett. B 716, 1 (2012) [arXiv:1207.7214].

[29] CMS Collaboration, S. Chatrchyan et al., Phys. Lett. B 716, 30 (2012) [arXiv:1207.7235].

[30] P. Gondolo, J. Edsjö, P. Ullio, L. Bergström, M. Schelke and E.A. Baltz, J. Cosmo. Astropart. Phys. 07, 008 (2004) [astro-ph/0406204].

[31] K. Griest and D. Seckel, Phys. Rev. D 43, 3191 (1991).

[32] S. Mizuta and M. Yamaguchi, Phys. Lett. B 298, 120 (1993).

[33] J. Edsjö and P. Gondolo, Phys. Rev. D 56, 1879 (1997).

[34] J.R. Ellis, T. Falk and K.A. Olive, Phys. Lett. B 444, 367 (1998); J.R. Ellis, T. Falk, K.A. Olive and M. Srednicki, Astropart. Phys. 13, 181 (2000).

[35] T. Nihei, L. Roszkowski and R. Ruiz de Austri, J. High Energy Phys. 0207, 024 (2002). 


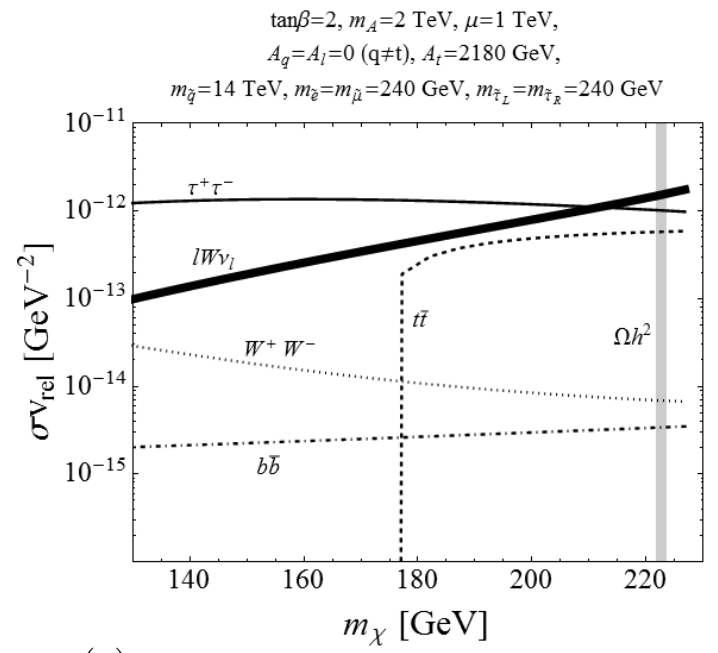

(a)

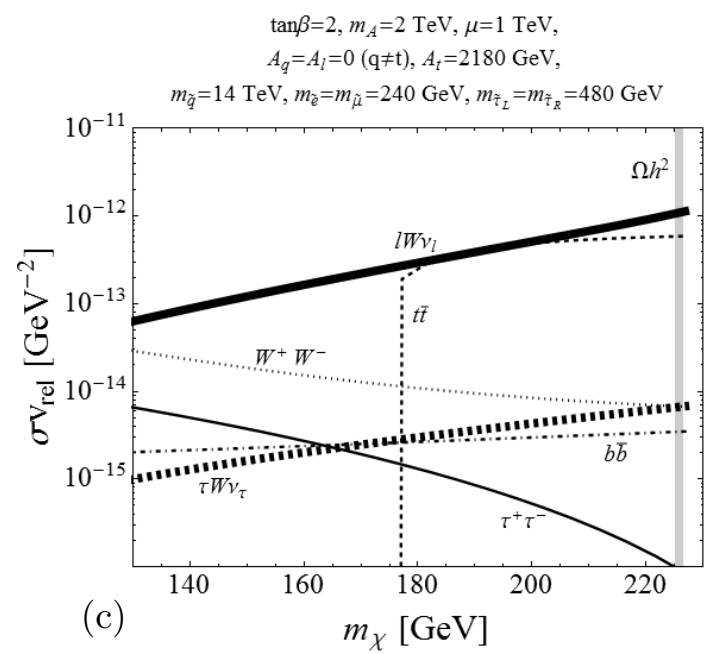

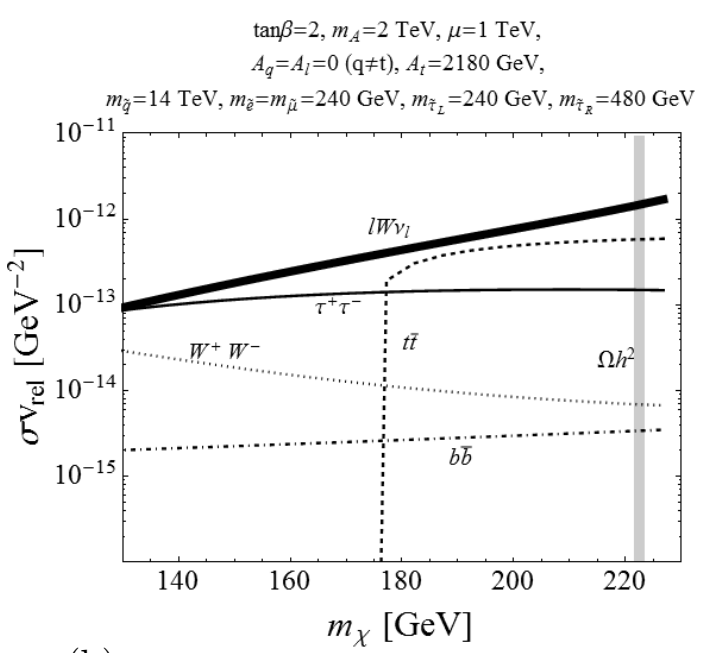

(b)

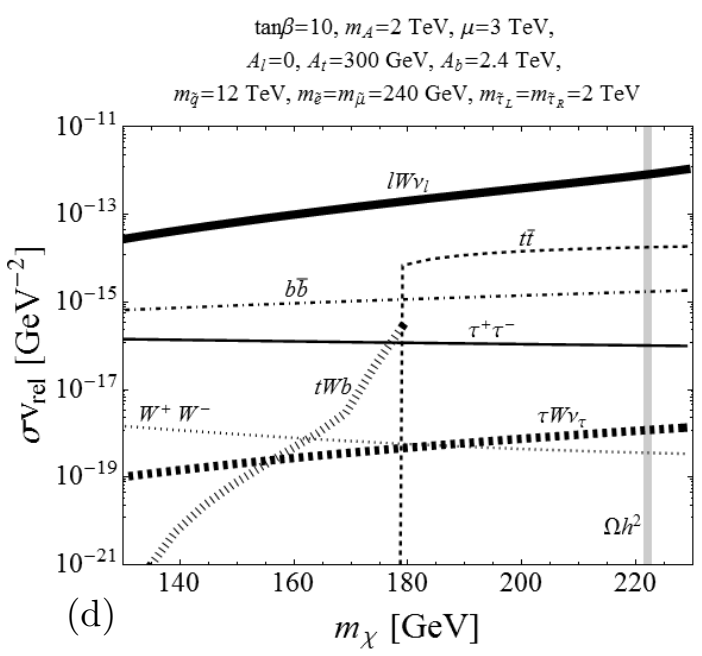

FIG. 2: (a) The cross section times the relative velocity, $\sigma v$, for the weak bremsstrahlung processes $\chi \chi \rightarrow \sum_{\ell} W \ell \nu$ (bold solid line), including both $W^{+} \ell^{-} \bar{\nu}_{\ell}$ and $W^{-} \ell^{+} \nu_{\ell}$, as a function of $m_{\chi}$. In panels (b), (c) and (d), the result for only $W \tau \nu_{\tau}$ is shown with the bold dashed line. The bold dotted line represents the contribution of $W t b$ evaluated with only $t$ - and $u$-channel diagrams. The contributions of the relevant two-body processes are shown with thin lines. The solid, dashed, dot-dashed and dotted lines correspond to $\tau^{+} \tau^{-}, t \bar{t}, b \bar{b}$ and $W^{+} W^{-}$, respectively. The range of $m_{\chi}$ filled with gray corresponds to the cosmologically allowed region where the relic abundance constraint $0.11<\Omega_{\chi} h^{2}<0.13$ is satisfied. 

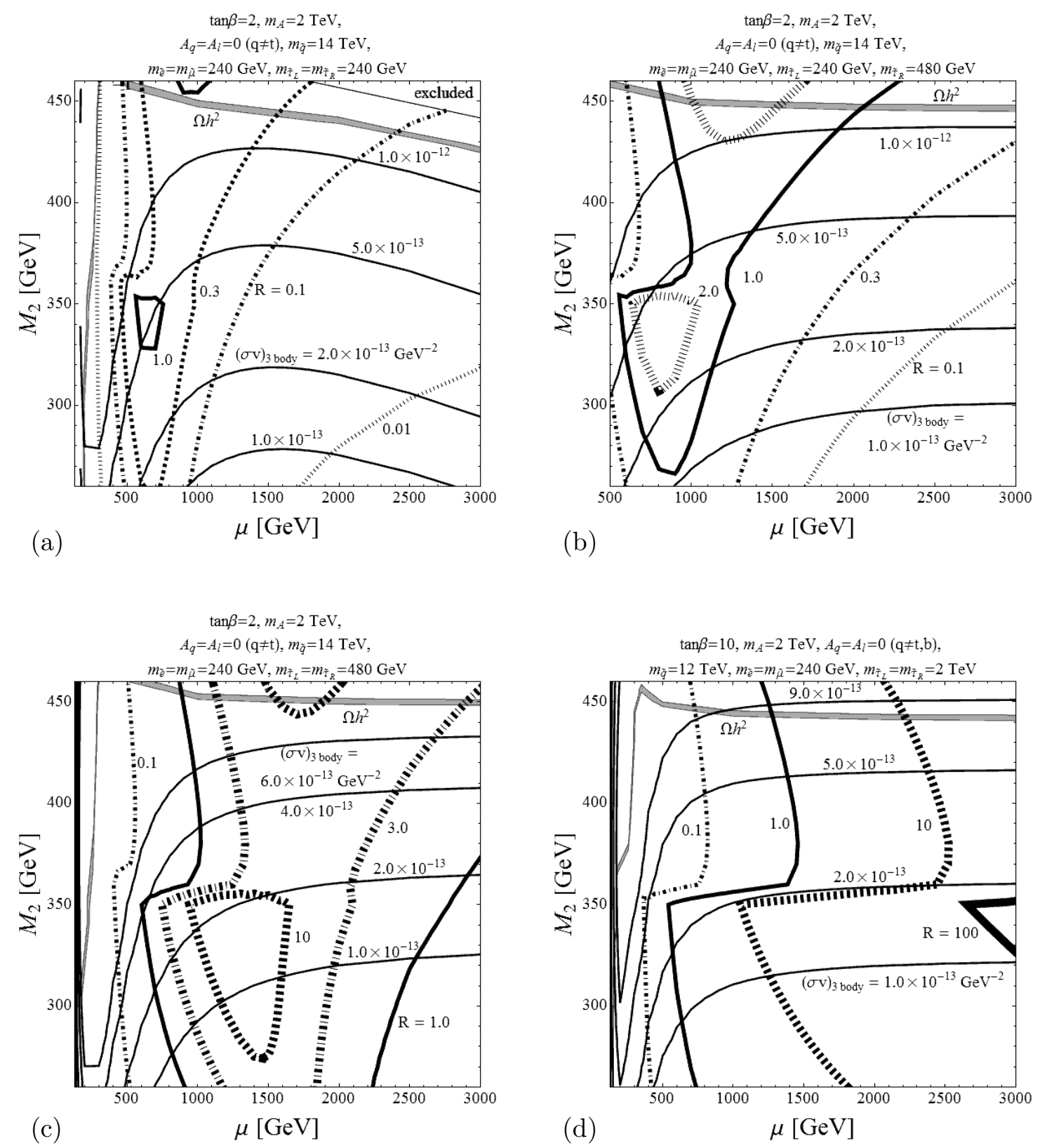

FIG. 3: Contours of the total $W$-strahlung contribution $(\sigma v)_{3 W}$ (thin solid lines). Contours of the ratio $R=(\sigma v)_{3 W} /(\sigma v)_{2}$ are shown in bold lines, where $(\sigma v)_{2}$ is the sum of all the two-body contributions. The strip filled with gray corresponds to the cosmologically allowed region with the correct $\Omega_{\chi} h^{2}$. 

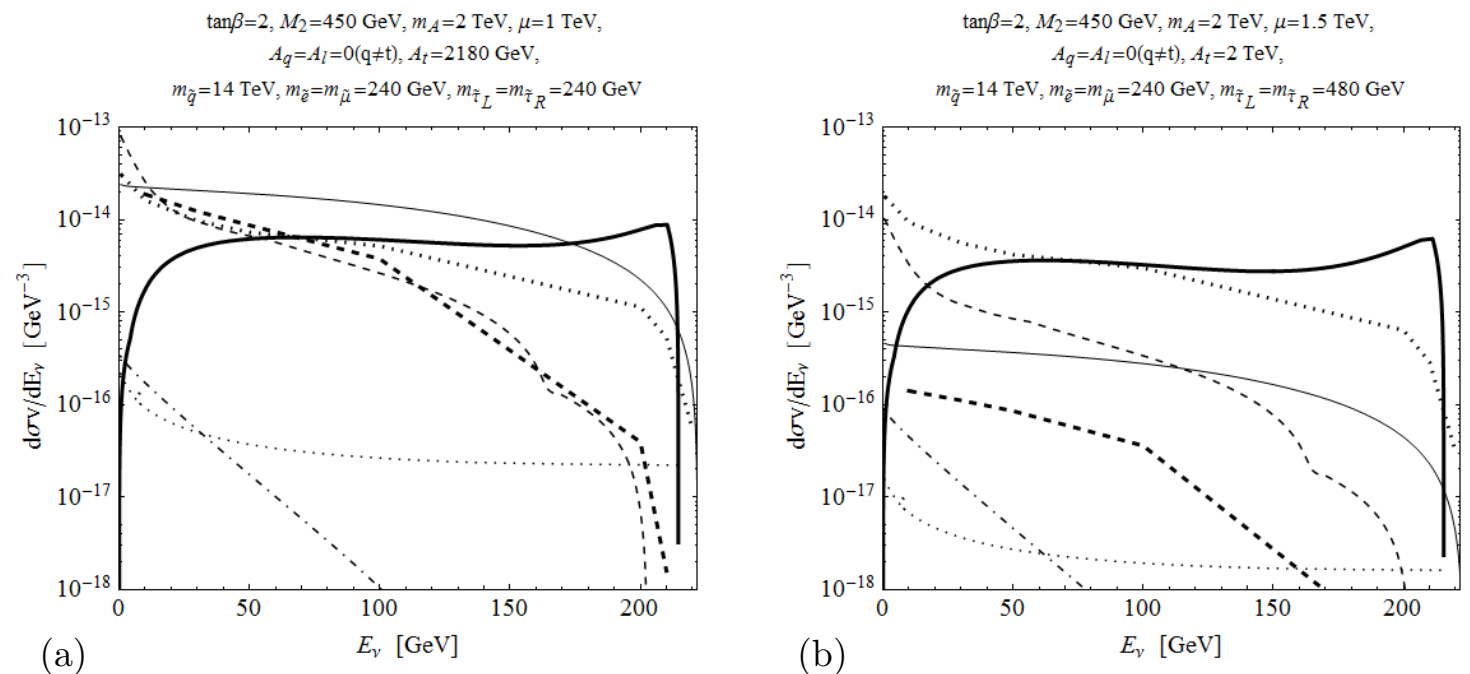

FIG. 4: Neutrino spectra at injection from the center of the Sun. The bold solid line corresponds to the primary neutrino spectrum of weak bremsstrahlung including all the flavors and the charge-conjugated states. The bold dashed and dotted lines are the results for the secondary neutrinos from the tau lepton and the $W$ boson, respectively, produced via weak bremsstrahlung. The contributions of two-body processes are drawn with thin lines. 